\title{
Listening to music on headphones during operations under regional anaesthesia
}

C. Harris ${ }^{1}$, T Bottomley $^{1}$, D. Kamming ${ }^{1}$

${ }^{1}$ Anaesthetic Department, University College London Hospitals (UCLH), London, United Kingdom

\section{Background}

- UCLH provides a dedicated "block room" for regional anaesthesia (RA)

- Preliminary data showed $50 \%$ of patients were anxious/very anxious

- $2 / 3$ were satisfied with their care overall

- Anxiety is often the reason for GA/sedation request on top of RA

- RA known to reduce post op pain and increase surgical pathway efficiency ${ }^{2}$

\section{How to improve?}

- Hole et al (Lancet 2015)1- "Music as an aid for postoperative recovery in adults"- found patients had less pain, less anxiety and more satisfaction

- Friends of UCLH charity grant- over $€ 1800$ awarded

- Enough for 10 sets

- Proposal presented to departmental CG meeting

- Ideas and concerns raised

- Implemented changes into plan

- Clearance sought from infection control

-Advice and standards established, approval gained

-Chlorhexidine wipes to headphones after each use

-10 sets of Sennheiser PXC 480 (noise cancelling) headphones purchased - $45 \%$ discount on RRP after negotiation

- Safety testing to ensure no interference with medical devices and compatible with diathermy use

- Patient engagement- raise awareness of service, request to bring in a device for playing music

- Logistics- storage and traceability

-Training- use and cleaning

-Re-audit

Figure 1. IMPLEMENTATION TIMELINE

\section{Methods}

- UCLH charity enabled us to procure 10 sets of noise cancelling headphones

- Implementation included clinical governance, infection control and medical physics approval (see fig. 1)

- Headphones offered to all patients who were undergoing RA

- Patients' satisfaction and feedback collected via google questionnaire before and after implementation of headphones. A visual analogue scale was used to assess anxiety

\section{Results}

- A total of 85 patients completed our questionnaire, 58 in the non-headphone group (NH) and 27 in the group which was provided with headphones $(\mathrm{H})$. $64 \%$ were female in the $\mathrm{NH}$ group compared to $56 \%$ in the $\mathrm{H}$ group

- On the visual analogue scale for anxiety, the patients with the headphones had a score of $4.7 / 10$ while the NH group had a score of 3.6

- In regards to the environment, all patients in the $\mathrm{H}$ group found it either relaxing or very relaxing. However, $9 \%$ in the $\mathrm{NH}$ group found it either stressful or very stressful

- Both groups showed similar levels of satisfaction$95 \%$ in the $\mathrm{NH}$ group and $96 \%$ in the $\mathrm{H}$ group

- $100 \%$ of the $\mathrm{H}$ group would recommend the UCLH regional anaesthesia service to friends and family. In the NH group, $12 \%$ would not

\section{Conclusion}

- Music has been shown to be beneficial for surgical patients in terms of anxiety and experience

- Offering patients noise cancelling headphones and music for RA is a simple, cheap and effective intervention and we showed it did improve overall experience

- Further research could look into the timing of music, and whether there is any improvement when given while waiting for surgery, during or in the post op period

- Other modalities such as virtual reality (VR) goggles should also be considered and evaluated

\section{References}

1. Hole J et al. Music as an aid for postoperative recovery in adults: A systematic review and meta-analysis. Lancet 2015 Aug 13; [e-pub]. (http://dx.doi.org/10.1016/S0140-6736(15)60169-6)

Kessler et al. regional anaesthesia and outcome: lessons learned from the last 10 years, BJA: British Journal of Anaesthesia, Volume 114, Issue 5, 1 May 2015, Pages 728-745, https://doi.ora/10.1093/bja/aeu559

- A short coming of this study is that the questionnaire was not designed to look at anxiety as the primary 